\title{
STRUCTURES D'ORGANISATION ET MOUVEMENT DES EFFECTIFS DU PARTI COMMUNISTE FRANÇAIS ENTRE LES DEUX GUERRES ${ }^{1}$
}

LES TYPES SUCCESSIFS D'ORGANISATION DU P.C.F.

Jusqu'en 1924, du point de vue de sa structure interne - la structure fédérale traditionnelle -, le Parti communiste français, bien que déjà section française de l'Internationale communiste, ressemblait, tel un fils à son père, au Parti socialiste unifié d'avant la scission. A son zème congrès national de Lyon (20-23 janvier 1924), tous les termes anciens sont encore en usage: à l'échelon local, la section rassemble les adhérents d'un même village ou d'une même ville (à Paris, d'un même arrondissement); la fédération englobe toutes les sections d'un même département; enfin, à l'échelon national, les délégués des 90 fédérations départementales, réunis annuellement en congrès, élisent un Comité directeur qui, s'appuyant sur des sous-commissions, a la responsabilité de la vie du Parti entre deux congrès.

Mais en juillet 1924, le sème congrès mondial de l'Internationale communiste réuni à Moscou décide, dans une résolution spéciale, que la section française devra procéder à un bouleversement complet de ses structures avant le rer janvier $1925:^{2}$ ce bouleversement seul étant de nature à la transformer d'un parti de type social-démocrate en un parti de type bolchevik. D'où le titre général de «bolchevisation» dont on baptise l'opération.

En quoi consiste-t-elle?

\section{La bolchevisation}

Elle repose essentiellement sur deux principes: il s'agit d'abord de modifier l'assiette sur laquelle repose la pyramide des organisations. La circonscription traditionnelle dans laquelle s'inscrit une section

1 Cette étude fait suite à une première analyse sur le mouvement des effectifs globaux du P.C.F. de I920 à 1939, parue dans la Revue française de sciences politiques, $n^{\circ}$ I, 1966 , pp. 5-35.

${ }^{2}$ Cf. Internationale Communiste, se congrès mondial. Résolutions [Petite bibliothèque communiste], Paris, l'Humanité $19^{24}$, in $8^{\circ}, 108 \mathrm{pp}$. 
socialiste, c'est le champ des luttes électorales dans un régime parlementaire bourgeois: la commune. La nouvelle circonscription dans laquelle doit s'inscrire l'organisation de base du Parti communiste à laquelle on donnera le nom de cellule, c'est le champ de bataille de la lutte des classes: l'usine, l'entreprise au sens large. Il s'agit ensuite, au nom de la conception bolchevique du centralisme démocratique, de renverser le primat traditionnel de l'autonomie des organismes inférieurs par rapport aux organismes supérieurs et de lui substituer une hiérarchisation rigoureuse qui permette aux organismes supérieurs d'avoir pleine autorité sur les organismes inférieurs.

Le Comité directeur du Parti communiste français approuvait le 20 juillet 1924 la résolution du sème congrès mondial sur la section française et celle-ci était le même jour publiée dans l'Humanité. Dès lors, les dirigeants français allaient déployer une intense activité: après que les sections locales aient été dissoutes, il leur fallut répartir les adhérents en fonction de leur lieu de travail et les y faire se constituer en cellules; regrouper les cellules dans un cadre nouvellement institué: les rayons; enfin regrouper les rayons dans un cadre également nouveau: les régions qui, au nombre de 27 seulement, se substitueraient aux 90 anciennes fédérations.

Ce branle-bas général devait soulever bien des difficultés et des querelles. Dans les premiers mois qui suivirent la réforme, l'incompréhension fut générale. Tantôt, hors des régions industrielles telles que la Région parisienne, la Région lyonnaise ou celle du Nord, on se contenta d'appeler cellules les anciennes sections. ${ }^{1}$ Tantôt au contraire on supprima la section sans se soucier de ce qu'allaient devenir les adhérents artisans, commerçants, ménagères qui ne pouvaient rejoindre une cellule d'entreprise. ${ }^{2}$ Il fallut d'ailleurs sur le tas apporter un certain nombre de correctifs secondaires au dispositif initial de façon à répondre à certaines situations concrètes qui n'avaient pas été prévues: c'est ainsi que la sème session du Comité exécutif élargi de l'Internationale dut décider qu'à côté des cellules d'entreprise proprement dites on pourrait créer des cellules de rue. ${ }^{3}$

Mais peu importent ici les péripéties et les modalités de cette «révolution» en matière d'organisation. Seules comptent pour notre propos les trois observations suivantes:

1 Cf. la "circulaire de la commission centrale d'organisation» publiće dans les Cahiers du bolchevisme, is mars 1925, p. 977. Cf. également le Rapport moral du Comité central, présenté par Pierre Semard au sème congrès national de Lille (19-26 juin 1926), in-fol., $30 \mathrm{pp}$.

${ }^{2}$ Id. Cf. également la discussion au 4ème congrès de Clichy, 17-2 I janvier 1925.

3 C.E.I.C. (Comité exécutif de l'Internationale communiste), sème conférence de l'Exécutif élargi (25 mars-6 avril 1925). Thèses et résolutions adoptées, Paris, l'Humanité, 1925, in-8, $64 \mathrm{pp}$. 
I0 - La «bolchevisation» entraîna un changement presque immédiat de la composition du Parti: «Notre parti, déclara P. Semard dans le rapport moral qu'il présenta au nom du Comité central à Lille, se renouvela dans la proportion de $70 \%$ de ses effectifs.» Beaucoup des hommes qui entretenaient dans le Parti communiste la vieille tradition socialiste le quittèrent à cette occasion. D'autres, formés dans la seule tradition bolchevique, les remplacèrent.

$\mathbf{2}^{\mathbf{0}}$ - Les principes d'organisation qui avaient sous-tendu l'opération de «bolchevisation» et qui trouvèrent leur expression dans les Statuts approuvés par le Bureau d'organisation du Comité exécutif de l'Internationale le 4 mai $1925^{1}$ ne furent jamais plus remis en cause: le fait que les communistes devaient dans toute la mesure du possible être adhérents d'une cellule d'entreprise a incontestablement joué un rôle ultérieur considérable, par exemple dans la conjoncture de juin 1936.

$3^{0}$ - La nouvelle structure du parti demeura, elle aussi, nonobstant des modifications de vocabulaire, ${ }^{2}$ intangible dans ce qu'elle avait d'essentiel.

${ }^{1}$ Cf. les Cahiers du bolchevisme, is juillet 1925 , p. 1485. Cf. l'Organigramme ci-joint.

2 Modifications au demeurant bien intéressantes et significatives. Les problèmes de vocabulaire sont, en effet, pour l'histoire du communisme français, d'une richesse particulière. D'abord parce que le mouvement communiste a secrété un langage qui lui est propre et qu'il faut connaître pour interpréter correctement ses publications. On sait que toute société fermée, ou même seulement minoritaire à l'intérieur d'une société plus large, a tendance, pour se différencier et consolider son homogénéité interne, à secréter, sinon un vocabulaire, du moins des sens spécifiques, des tournures, des constructions de phrase qui constituent un véritable code de reconnaissance. En outre une société qui, sans être secrète, veut protéger ses "secrets» contre la curiosité d'un monde extérieur jugé globalement hostile, a également tendance à se prémunir par l'usage d'un code que les affidés seuls savent transcrire: dans les conférences de rayon ou de région, un chapitre était souvent consacré au «travail anti». Abréviation passée dans le langage courant pour désigner le «travail antimilitariste», illégal ou semi-illégal. C'est ce double processus, mispontané, mi-organisé, qui a produit le ulangage» communiste. Par exemple, une phrase comme celle-ci: «Développer notre travail anti de masse sur la base du plan régional et en accord avec les J.C.» (Rapport politique à la conférence de la Région parisienne, fév. I932, p. 28) est incompréhensible au non-initié.

Autre exemple: en matière d'effectifs. Dans les années 30 , on parlait de retard pour désigner une chute considérable du nombre des adhérents: ce terme lénifiant avait spontanément surgi du fait que la reprise des cartes s'effectuant massivement en janvier $m z$ is pouvant ensuite s'étendre et se prolonger sur les mois suivants, ne pas atteindre les effectifs de l'année précédente pouvait ne constituer qu'un retard. Le terme de tassement (des effectifs) désignait également un recul des effectifs, sensible mais moindre que dans le cas d'un retard. Enfin celui de consolidation (toujours des effectifs) était employé pour caractériser une situation où retard et tassement auraient été l'un et l'autre trop pessimistes, mais où les signes d'un nouvel essor n'apparaissaient pas encore.

Mais l'étude du vocabulaire communiste n'est pas seulement intéressante comme contribution à la compréhension des textes et documents officiels; elle peut permettre de 


\title{
ORGANIGRAMME \\ DU PARTI COMMUNISTE FRANÇAIS \\ ENTRE I925 E'T 1939
}

\begin{abstract}
Comité exécutif de l'Internationale communiste
Elu par le congrès de l'Internationale communiste composé des delégués des Partis communistes des divers pays. Le Comité exécutif de l'Internationale communiste dirige entre les congrès de l'Internationale communiste l'activité de tous les Partis communistes.
\end{abstract}

Secrétariat - Bureau politique ...... élu par le Comité central

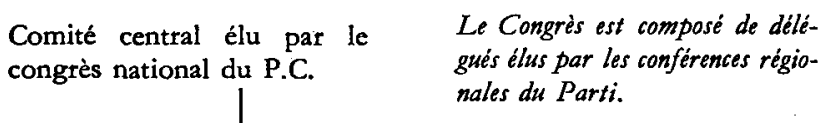

Comité central élu par le<smiles>C=[V]</smiles>

La conférence régionale est com- Comité régional élu posée des délégués élus par les par la conférence conférences de rayons.

Bureau de rayon régionale<smiles>[CH]1[CH]CC1</smiles>

Comité de rayon<smiles>[Li][14CH2]</smiles>

Bureau régional

Elu par la conférence de rayon composée des délégués élus par les assemblées de cellule

Bureau de sous-rayon

Elupar l'assemblée de tous les membres communistes du sousComité de sous- rayon rayon

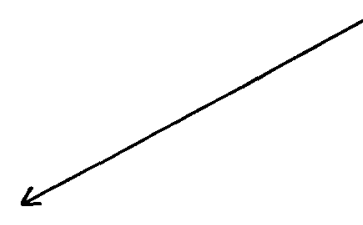

Céllule de rue

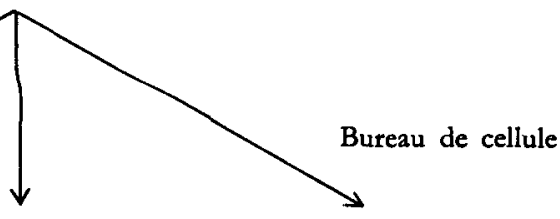

Cellule d'entreprise
Cellule de village

Réf. P.C. (S.F.I.C.), Au nouvel adhérent du P.C. Int. de Jacques Duclos, Paris, Bureau d'Editions, 1932, in-16, 16 pp. 
Le Comité central du P.C.F. réuni en novembre I924 avait donc décidé de resserrer les 90 fédérations en 27 régions. Le dessin de ces Régions donna lieu à d'âpres marchandages entre le Centre et les militants locaux. ${ }^{1}$ Ceux-ci en effet arguaient des impératifs commerciaux de la presse régionale communiste, de la disposition des moyens de communication, des affinités traditionnelles entre pays de même vocation agricole pour obtenir un découpage qui respectât au maximum les habitudes locales. Au contraire le Centre avait mission de prendre pour seul critère la présence de zones industrielles et de concevoir des régions dont le cœur serait constitué par des villes usinières auxquelles serait accolé du plat-pays agricole.

Huit ans plus tard, en 1932, la carte des Régions du Parti s'est un peu modifiée. Mais en octobre 1932 le Comité central qui cherche

fixer la chronologie des modifications introduites dans la stratégie communiste. On sait par exemple avec quelle insistance l'Internationale communiste a exigé des anciens partis socialistes qu'ils changent leur dénomination dès lors qu'ils décidaient d'adhérer à la Troisième Internationale. La I 7 ème des 21 conditions d'adhésion à l'Internationale stipulait en effet: "Tout parti désireux d'adhérer à l'I.C. doit s'intituler: Parti communiste de ... (section de la IIIeme Internationale communiste).» Plus tard, la «bolchevisation» du Parti se traduisit par une bolchevisation et par conséquent une russification des termes désignant les diffétents niveaux de l'appareil: alors que l'ancienne pyramide était formée de sections, fédérations et du comité directeur (termes issus de la tradition politique française et singulièrement de la jacobine), la nouvelle pyramide fut formée de cellules, rayons, régions et du comité central (termes empruntés à la hiérarchie du parti bolchevik). L'histoire la plus curieuse est celle du mot rayon. En russe, le raion, qui est lui-même sans doute un gallicisme, désigne un district, un arrondissement. Le "rayon" fut donc bien a l'origine une circonscription mineure dans la hiérarchie des organisations communistes françaises. Mais, assez vite, il se fit dans la tête des militants communistes français une confusion entre le rayoncirconscription et le rayon (de roue), d'où l'idée de partager la Région parisienne en rayons ayant la forme des espaces triangulaires entre deux rayons de roue.

La transposition de termes et de tournures russes (abréviations) dans l'organisation du P.C.F. constitua une véritable mode dans les années 1924-1934: on parlait d'agit-prop, du bureau d'org. etc. Cela dura tant que la stratégie d'isolement «classe contre classe» fut maintenue. Mais quand en 1935-1936 la stratégie du Front populaire puis du Front français conduisent le P.C.F. à remettre l'accent sur les valeurs nationales, la russification du vocabulaire communiste fut abandonnée. C'est le 23 janvier 1937 que la conférence nationale du P.C.F. décide de reprendre le terme de section pour désignet ce qu'on appelait rayon, "dénomination plus simple et correspondant mieux aux formes traditionnelles d'organisation existant dans notre pays". Il y eut donc désormais à nouveau côte à côte une section communiste et une section socialiste: mais, ultime nuance, les communistes parlaient de la section du XIVème (arrond.) tandis que les socialistes parlaient de la XIVeme section! Désormais l'utilisation de termes russes tels que Komintern pour Internationale communiste, Inprekorr pour Correspondance internationale fut le fait d'adversaires entendant donner le parti communiste pour un parti inspiré de l'étranger ou de communistes de la vieille génération ne pouvant se défaire de leurs anciennes habitudes de langage.

1 C. Cahiers du bolchevisme, r925. 
désespérément un remède à la crise du recrutement décide de «décentraliser» les Régions

«dont les rayons, trop vastes ne permettent pas un contact permanent du Parti avec la classe ouvrière, ni des directions avec les organisations de base; cela en vue de développer la vie politique et l'activité des organisations du Parti, d'élargir notre cadre de militants, d'établir les bases d'un véritable travail collectif, de créer et développer l'esprit de responsabilité en même temps que l'initiative de chaque membre du Parti. Le Comité central décide de commencer cette décentralisation dans la Région parisienne.» ${ }^{1}$

Effectivement la Région parisienne qui groupait les trois départements de la Seine, de la Seine-et-Oise, de la Seine-et-Marne donne naissance à cinq Régions dont la structure est toujours fondée sur l'idée qu'une région doit avoir un cour industriel et des dépendances agricoles. A l'exception de la Région Paris-Ville qui correspond aux 20 arrondissements de la capitale, les quatre autres Régions (ParisNord, Paris-Ouest, Paris-Sud, Paris-Est) comprennent des communes de banlieue du département de la Seine et de la Seine-et-Oise et des communes agricoles (le département de la Seine-et-Marne revenant tout entier à la Région Paris-Est).

En octobre 1934, l'effort de décentralisation a porté le nombre des Régions à 46. Mais le renversement de la conjoncture et l'afflux des adhérents contraignent à le pousser plus loin: le I I octobre 1935, le nombre des régions est de 60; en 1936, de 68: cette année-là sont créées les nouvelles Régions de la Corse, de la Côte d'Or, de la Moselle, du Pas-de-Calais, du Puy-de-Dôme, du Tarn-et-Garonne, du Vaucluse, de l'Yonne; en 1937 on atteint le chiffre de 72: la Région algérienne du P.C.F. disparaît pour faire place au Parti communiste algérien; par contre cinq régions nouvelles sont créées: du Lot-etGaronne - Gers, de la Marne, du Var, des Vosges, du Territoire de Belfort.

Ainsi la poussée de croissance des années I934-1938 a eu pour conséquence de conduire à calquer à nouveau les régions communistes sur les anciennes fédérations départementales socialistes.

\section{Les rayons}

Dans l'échelle hiérarchique, les rayons constituent l'échelon intermédiaire entre la région et la cellule (encore que, avec l'afflux des

${ }^{1}$ P. C. de France (S.F.I.C.), Quatre années de lutte pour l'unité, le pain, la paix, la liberté (VIIIle congrès national, 22-25 janvier 1936), p. 65. 
adhérents à partir de 1935 , il sera nécessaire de constituer pour renforcer l'armature un niveau supplémentaire entre le rayon et la cellule: le sous-rayon).

Au début quand ils sont créés dans les années $1925-1926$, le territoire des rayons est conçu de façon à «faire correspondre l'organisation du parti aux divisions administratives de l'Etat bourgeois.» ${ }^{1}$ Dès lors en effet que les cellules - les organisations «opératives» au contact direct des masses ouvrières - sont en priorité établies sur la base des entreprises, il n'y a aucun inconvénient à ce que le niveau supérieur, ayant surtout vocation d'administration interne, puisse, le cas échéant, prendre toutes mesures nécessaires à l'organisation des combats électoraux - dont le Parti communiste français, dans la ligne des décisions du zème congrès de l'Internationale, ne s'est jamais exclu, à la différence des anarchistes ou des ultra-gauches françaises et européennes des années 20.

Calqués sur les circonscriptions administratives de l'appareil d'Etat, les rayons sont découpés de telle manière que leur comité puisse effectivement contrôler l'ensemble des cellules implantées sur toute l'étendue de leur territoire. Le type de circonscription administrative choisie pour y faire correspondre un rayon est en conséquence fonction de la densité des cellules. Il y a finalement deux catégories de rayon: $\mathbf{I}^{0}$ Les rayons de ville (Bordeaux, Marseille) ${ }^{2}$ qui ont autorité sur toutes les cellules d'une même ville; $2^{0}$ Les rayons de province dont le territoire correspondra à un canton, à un arrondissement et même à un département quand la densité des cellules qui y sont implantées est particulièrement faible.

Le nombre de rayons établis dans une même région n'est d'ailleurs pas rigide. Si l'on compare le nombre de rayons et le nombre de régions pour les années 1932, 1934, 1935, 1936, 1937, on s'aperçoit que le nombre moyen de rayons par régions est en augmentation.

$\begin{array}{cccc}\text { Années } & \text { Régions } & \text { Rayons } & \text { Moyenne } \\ 1932 & 29 & 200 & 6,8 \\ 1934 & 46 & 300 & 6,5 \\ 1935 & 60 & 445 & 7,4 \\ 1936 & 68 & 834 & 12,4 \\ 1937 & 72 & 976 & 13,5\end{array}$

De même, le nombre de cellules contrôlées par un même comité de rayon n'est pas fixe: dans tel rayon de ville, le nombre de cellules

1 P.C.F., sème congrès national (Lille, 20-26 juin 1926). Compte rendu sténographique, p. 670 .

2 Paris est un cas particulier. 
pourra atteindre une vingtaine $;^{1}$ dans tel rayon de province, il pourra descendre à quatre. ${ }^{2}$

\section{Les cellules}

Rayons et régions - en 1936 sections, en I94s fédérations - sont les dénominations choisies par le Parti communiste pour désigner les échelons intermédiaires dont tout appareil ramifié a besoin pour permettre des communications aisées entre le centre et sa base. Les cellules au contraire constituent des organes tout à fait originaux dont l'équivalent n'existe pas dans les autres partis, notamment le Parti socialiste.

Le nombre total de cellules constituées n'est pas à lui seul indicatif de l'importance globale des effectifs à un moment donné, car le nombre d'adhérents par cellule n'est pas rigide. ${ }^{3}$ Le Centre a poussé parfois à la «décentralisation» non parce que le chiffre des membres d'une même cellule était devenu en soi trop élevé, mais au contraire parce que le déclin de ses effectifs semblait dû au fait qu'elle avait la responsabilité d'une circonscription trop vaste: ce qui était alors tenu pour excessif, c'était, selon le vocabulaire en usage, son «territoire». Il est arrivé en revanche - car les décisions de "centralisation» et de «décentralisation» alternant régulièrement ont souvent joué dans le P.C.F. le rôle que joue ailleurs l'institution de commissions: c'est un remède universel et sans danger aux difficultés de l'heure - qu'on «centralise» deux cellules dont chacune était trop squelettique pour avoir une activité efficace. C'est ce qui explique qu'en 1929, le total du nombre des cellules était de $3 \cdot 308,{ }^{4}$ alors qu'il n'était plus que de 2.725 en octobre $1934 .^{5}$

Cependant la progression du nombre des cellules entre 1935 et 1938 n'est pas fortuite: en juin 1935 , celui-ci était de 3.647 cellules. ${ }^{6}$ En

\footnotetext{
1 Exemple: la Région du Nord a en 1925300 cellules groupées en 17 rayons. Moyenne: 17 cellules par rayon.

2 Exemple: la Région Nord-Est a en 192589 cellules pour I9 rayons. Moyenne: 4 cellules par rayon.

${ }^{3}$ En 1928, la moyenne du nombre d'adhérents par cellule était de 13,33 . En 1930, dans le sous-rayon du XIXème arrondissement de Paris, 3 cellules de rue avaient respectivement 16, 17 et 18 adhérents (Le Carnet du Militant, $n^{0}$ ).

4 P.C.F., Congrès national (3 I mars-6 avril 1929). Rapport politique du Comité central, Paris, Bureau d'éd., I929.

5 P.C.F., Deux ans d'activité au service du peuple ..., p. Io2 (gème congrès national, 25-29 déc. 1937).

6 M. Gitton, Le parti des travailleurs de France, p. 13 . Rapport présenté au VIIIème congrès national du P.C. (S.F.I.C.), Villeurbanne, 22-25 janvier 1936.
} 
octobre 1935, de 4.221. ${ }^{1}$ En octobre 1936, de 10.736. ${ }^{2}$ En octobre 1937, de $12.992 . .^{3}$ En octobre 1938 , de $12.6544^{4}$

Mais le chiffre total de cellules sans distinction de catégorie ne saurait rendre exactement compte de la nature de l'enracinement du Parti communiste français à un moment donné. En effet, on l'a vu, il existe plusieurs types de cellules dont l'un est privilégié: celui auquel correspondent les cellules d'entreprise.

Déjà au zème congrès mondial de juin I92I, le Komintern lançait le mot d'ordre: "Allez aux masses!», et à son 4ème congrès mondial en novembre 1922 recommandait de créer des cellules sur les lieux de travail. Mais c'est au sème congrès mondial qu'en juillet 1924, dans le cadre d'une stratégie d'attente à laquelle était liée la «bolchevisation des Partis communistes», le tournant en matière d'organisation était véritablement pris. ${ }^{5}$ Après que le congrès lui-même en aît posé les principes, ${ }^{6}$ le Comité exécutif de la Troisième Internationale dut réunir deux conférences successives d'organisation, la première du I6 au 21 mars $1925,{ }^{7}$ la seconde en mars $1926 .{ }^{8}$ Celle-ci adopta notamment de volumineuses «Instructions sur la structure et le fonctionnement des Cellules d'entreprise et de rue» ${ }^{9}$ qui passaient minutieusement en revue les difficultés et les problèmes soulevés.

${ }^{1}$ Deux ans d'activité au service du peuple ..., p. Io2. Chiffre un peu incertain: Marcel Gitton, au congrès d'Arles en décembre 1937, donne le chiffre de 4.321. Par ailleurs le total calculé à partir des déclarations faites par les régions donne le chiffre de 4. I 18 . Il ne faut pas oublier que, si le nombre de régions et de rayons n'est pas soumis à d'autres fluctuations que volontaires et décidées d'en haut, le nombre des cellules au contraire est soumis à des aléas venant d'en bas: il suffit souvent que le secrétaire d'une petite cellule de village ou de rue déménage, que le secrétaire d'une petite cellule d'atelier soit licencié pour que la cellule disparaisse ou, du moins, entre en sommeil. Les cellules sont des organismes fragiles: d'où la difficulté de les comptabiliser avec précision.

2 Deux ans d'activité au service du peuple ..., p. Ioz.

s Id.

4 Une année de lutte .., p. 123.

5 Pour une brève information sur l'histoire de la Troisième Internationale, cf. A. Kriegel, Les Internationales ouvrières (1864-1943), Paris, P.U.F., I964.

- Cf. Internationale Communiste, Les questions d'organisation au gème congrès de l'I.C. Cellules d'entreprises, statuts de l'I.C., directives pour l'organisation [L'I.C. et les questions d'organisation, $\mathrm{n}^{\circ} \mathrm{I}$ ], Paris, l'Humanité, I925, in-16, I04 pp. Cf. également P.C., Les cellules communistes d'entreprise [Les Cahiers du Militant, $\mathrm{n}^{0} 4$, sept. 1924], Paris, Librairie de l'Humanité, s.d., in-8, 26 pp.

7 Internationale Communiste, La réorganisation des P.C. Rapports et décisions de la Conférence d'organisation de l'I.C. (16-21 mars 1925) [L'I.C. et les questions d'organisation, $\mathrm{n}^{0}$ 2], Paris, Librairie de l'Humanité, 1925 , in-I6, $192 \mathrm{pp}$.

${ }^{8}$ Internationale Communiste, La seconde conférence d'organisation. Décisions et résolutions adoptées par la zème conférence du Comité exécutif de l'I.C. et ratifiées par le Bureau d'organisation du Comité exécutif de l'I.C. le 26 mars 1926 (Préface de J. Piatnitsky [L'I.C. et les questions d'organisation, $n^{\circ} 3$ ], Paris, Librairie de l'Humanité, 1926,in- $16,98 \mathrm{pp}$.

${ }^{\circ}$ Cf. Le Carnet du Militant, octobre $1928, \mathrm{n}^{\circ} \mathrm{I}$, publié par la Section centrale d'organisation 
La création en France de cellules d'usine se transforma en un combat plein d'héroïsme. «La section, reconnaissait Pierre Semard, c'était un peu loin du patronat, un peu loin du capitalisme, mais la cellule, c'est beaucoup plus près.» 1

En juin 1926, le sème congrès national réuni à Lille avait adopté des «Statuts» ${ }^{2}$ dont l'article I 3 précisait bien: «La cellule d'entreprise est la base de l'organisation du parti (à la fabrique, à la mine, à l'atelier, au bureau, au magasin, dans l'entreprise agricole, etc. ...).» Mais il ne suffisait pas de légiférer pour faire passer la chose dans la pratique. En 1927 , les cellules d'entreprise ne représentaient encore que $31 \%$ du nombre total des cellules existantes. ${ }^{3}$ En octobre 1928 , le Carnet du Militant soulignait dans son préambule sous le titre «Il faut lutter contre le révisionnisme d'organisation" que "la dernière conférence d'organisation a signalé dans les rangs de notre Parti une tendance à la désertion des cellules d'entreprise, au retour à l'organisation locale.» ${ }^{4}$ La même année, $O$. Piatnitski faisait état de 898 cellules d'usine groupant 17.448 communistes, ce qui représenterait d'après lui $33 \%$ des effectifs. ${ }^{5}$ Mais un recensement partiel ne fait apparaître pour I.I42 cellules locales et de rue que 358 cellules d'entreprise, soit $23,86 \%$ de l'ensemble: ces 358 cellules ne groupant au demeurant que 5.660 adhérents dispersés dans des usines où travaillent 290.105 ouvriers. ${ }^{6}$ En 1929 enfin, les cellules d'entreprise ne représenteraient encore que $29,2 \%$ du total. 7

$\mathrm{Au}$ surplus, une pratique s'était instituée qui, avec les meilleures intentions du monde, risquait de tourner purement et simplement les principes admis: la pratique des «rattachés» que Piatnitski dénonce en ces termes: "Ce qui saute aux yeux, c'est le grand nombre de communistes membres de cellules d'usine. Mais cela s'explique par le fait

sur le thème: «Cellules d'entreprise et cellules de rue (Structure, fonctionnement, tâches). Nouvelle édition des décisions adoptées à la zème conférence d'organisation du C.E. de l'I.C.», Paris, BUIC, s.d., in-16, 48 pp.

${ }_{1}$ P.C., sème congrès national (Lille). Compte rendu sténogr., p. Ix. Le ier mai 1926, il existait selon P. Semard (Humanité, 16/6/26), 1.544 cellules d'entreprise pour un total de 3.188 cellules.

${ }^{2}$ Cf. le texte des statuts adoptés par le congrès de Lille dans la brochure Statuts du Parti communiste français. Propositions de modifications. Avant-propos de Marcel Gitton, Bourges, Imp. ouv. du Centre, I9 pp. Ces statuts de I926, consacrant le «toumant" en matière d'organisation remplaçaient le Règlement adopté par le congrès extraordinaire des IsI7 mai I921 et modifié par le congrès national d'octobre ig23. Ils furent à leur tour modifiés une première fois en 1936 sur décision du congrès de Villeurbanne (janvier 1936).

${ }^{3}$ Rapport politique du Comité central, p. 98 (Gème congrès national, Paris, 1929).

4 Le Carnet du militant, oct. $1928, \mathrm{n}^{\circ} \mathrm{I}$.

5 O. Piatnitski, Quelques problèmes urgents ..., p. 26.

- Rapport politique du Comité central, 1929, p. 999.

7 Id., p. 98. 
que pour 3 membres travaillant vraiment dans l'usine, on en rattachait Is à 18 autres, de sorte qu'on ne peut pas dire qu'il y a vraiment une grande proportion de communistes travaillant dans les usines." ${ }^{1}$ Un sondage effectué en 1929 montrait en effet que parmi les adhérents des cellules d'entreprise, $21,15 \%$, soit plus d'un adhérent sur cinq, étaient des «rattachés», c'est-à-dire des communistes qui ne travaillaient pas effectivement dans l'usine où la cellule était en principe implantée, soit qu'ils fussent des fonctionnaires permanents du parti, ${ }^{2}$ soit qu'ils fussent des ouvriers de la même corporation que ceux travaillant dans l'usine en question mais perdus dans de petits ateliers où il n'y avait pas de cellule. ${ }^{3}$ Défalcation faite de ces «rattachés», seulement 22, 15\% des membres du Parti militent sur leur lieu de travail. ${ }^{4}$ Le même sondage révélait que $58,66 \%$ des métallurgistes membres du parti étaient adhérents à des cellules locales: moins d'un

\footnotetext{
${ }^{1}$ O. Piatnitski, op. cit., p. 26.

${ }^{2}$ Les «permanents», fonctionnaires appointés par le Parti, étaient généralement affectés à une cellule d'entreprise appartenant à la corporation dont ils étaient issus: par exemple, un ancien cheminot était affecté à une cellule de cheminots, etc.

sa répression, très aiguë dans les grandes entreprises, faisait se réfugier beaucoup de communistes dans de petits ateliers où ils retrouvaient une certaine "virginité». En principe, ils devaient y rester un certain temps, le temps que les employeurs aient perdu leur piste, le temps aussi d'obtenir un certificat de travail. Après quoi, ils devaient se faire réembaucher dans une grande entreprise où le travail politique et syndical était plus important. Mais, en période de chômage, il fallait beaucoup de courage pour prendre de tels risques et un certain nombre de militants se lassaient, refusaient de quitter la «petite boîte» où ils étaient tranquilles pout retourner dans la grande d'où, à plus ou moins longue échéance, ils seraient chassés. A noter que la mobilité ouvrière était beaucoup plus grande qu'aujourd'hui et qu'à Paris surtout, l'ouvrier qualifié (de la métallurgie) se faisait gloire de "demander son compte» dès qu'on lui cherchait noise.

4 Rapport politique du Comité central, I929, p. 98. Le Carnet du Militant n ${ }^{\circ}$ 5, donne un exemple encore plus précis: en janvier 1930, dans le sous-rayon du XIXème arrt., les choses se présentaient de la manière suivante:
}

\section{Cel. d'entreprise}

Richard (métal.)

Gaumont films

P.T.T.

Pompes funèbres

Abattoirs

Gaz

$\begin{array}{cc}\text { Nbre d'ouvr. } & \begin{array}{c}\text { Effectifs } \\ \text { cellule }\end{array} \\ 300 & 10 \\ 800 & 9 \\ 200 & 11 \\ 800 & 10 \\ 1.200 & 10 \\ 1.100 & 21\end{array}$

Travaillant
à l'usine
5
5
4
5
2
19

Rattachés

On remarquera que sur les 6 cellules d'entreprise du sous-rayon du XIXème arrt, 2 seulement sont des cellules d'usine; les 4 autres sont des cellules d'entreprises à statut: c'était là un autre point faible que les statistiques dissimulaient trop souvent. Il était en effet plus facile et moins dangereux de constituer des cellules dans les entreprises à statut (chemins de fer, gaz, électricité, P.T.T., mines) que dans les entreprises tout à fait "privées" de la métallurgie, des produits chimiques, etc. 
sur deux était donc affecté à une cellule d'usine. ${ }^{1}$ Cette pratique fâcheuse se poursuit, malgré les mises en garde: dans le is ème rayon de la Région parisienne en I93 I, 7 cellules d'usine comptaient I 2 adhérents travaillant effectivement à l'usine et 42 "rattachés».

Il faut ajouter que la structure de la profession (dans l'industrie du bâtiment par exemple, avec la mobilité des équipes liées provisoirement à l'existence de tel chantier), l'organisation du temps de travail (avec la succession des «équipes» de jour et de nuit ou des «brigades»), la composition de la main-d'œuvre (avec la pratique du "ramassage» dans le Textile, les Mines et certaines entreprises métallurgiques de province; avec la présence d'importants contingents d'ouvriers étrangers non francophones, etc.) posaient de difficiles problèmes d'organisation: une longue pratique permit seule de dégager les moyens de rassembler les communistes dans des entreprises aussi complexes que les Chemins de fer, la Marine de commerce ou parmi les travailleurs saisonniers de l'agriculture. Dans chaque cas, il fallut préciser l'unité qui constituerait le «territoire» de la cellule: atelier, bureau, chantier, etc. Mais la question la plus difficile et la plus mal résolue fut toujours celle de l'organisation des chômeurs.

Ces difficultés n'empêchaient pas que soit envers et contre tout maintenu le principe:

"C'est de l'usine, répétait on au «nouvel adhérent», de la mine, du chantier, du bureau que monte le cri de colère qui unit dans la même lutte tous ceux qui subissent la loi du capital et de l'exploitation de l'homme par l'homme. C'est à l'usine, à la mine, au bureau, au chantier que sont rassemblés par les capitalistes des milliers et des dizaines de milliers de travailleurs. L'usine, c'est le centre nerveux de la société moderne, c'est le foyer même de la lutte de classe. C'est pourquoi l'usine doit être pour toi, communiste, le centre de tes efforts, de ton activité de communiste.»²

Aussi, lorsque la conjoncture devint favorable, le nombre de cellules d'entreprise augmenta-t-il vertigineusement: si en octobre 1934, il n'était encore que de 586 , en juin 1935 , on comptait déjà 738 cellules d'entreprise; en octobre 1935, $776 ;^{3}$ en octobre 1936, 2.898; en octobre $1937,4.041 ;^{4}$ en septembre $1938,3.858 .5$

\footnotetext{
1 Rapport politique du Comité central, I929, p. 98.

2 P.C., Au nouvel adhétent, Préface de J. Duclos, p. s.

3 M. Gitton à Villeurbanne.

4 Id. à Arles.

5 P.C., Une année de lutte ..., p. 123.
} 
Cette analyse préalable des types successifs d'organisation du Parti communiste français va nous permettre, en ayant la certitude de comparer des chiffres comparables, de mesurer les inégalités de l'implantation communiste régionale.

\section{La densité communiste en 1936}

En prenant pour base les chiffres des cartes expédiées à la fin de l'année 1936 dans chaque région ${ }^{1}$ et on les rapportant aux chiffres correspondants de la population, ${ }^{2}$ on obtient la «densité» communiste régionale. ${ }^{3}$

Que celle-ci se situe entre 7 et 208 communistes pour 10.000 habitants démontre combien elle est inégale suivant les régions; mais le fait que sur les 64 régions métropolitaines, I 8 seulement avaient alors une densité supérieure à la densité nationale $(0,67 \%)$ démontre encore mieux l'extrême inégalité de l'implantation communiste en France.

En classant les régions en fonction de leur densité communiste, on en aperçoit nettement les lignes de force et de faiblesse: $\mathrm{r}^{0}$ - Les régions dont la densité communiste est supérieure á $75 \mathrm{com}$ munistes pour 10.000 babitants constituent trois blocs: la Région parisienne jusqu'à la frontière du nord (à l'exception du Pas-de-Calais, un peu plus faible), l'Est autour de la sidérurgie lorraine, le Sud-est de Marseille à la frontière italienne. Il faut ajouter à ces trois blocs trois départements où l'implantation communiste est également très forte: la Gironde, la Corrèze, les Pyrénées-Orientales.

$2^{0}$ - Si l'on ajoute à cette première catégorie les régions dont la densité communiste se situe entre so et $75 / 10.000$ babitants, on a alors la configuration exacte des lignes de force relative de l'implantation communiste: de la Région parisienne à la frontière du Nord, des Ardennes à la Lorraine, tout le pourtour de la Méditerranée avec une nette remontée le long de la vallée du Rhône, enfin le pourtour septentrional et occidental du Plateau central jusqu'à l'Océan.

$3^{0}$ - Les régions dont la densité communiste est inférieure à so/10.000

${ }^{1}$ En 1936, la différence entre cartes expédiées et cartes placées est peu sensible, du fait de la croissance rapide des effectifs. Les chiffres utilisés ici sont ceux de décembre 1936.

${ }^{2}$ Comme il n'était pas possible de choisir seulement le chiffre des électeurs puisqu'il ne comprenait pas en 1936 les femmes, c'est le chiffre de la population totale qui a été retenue. S'en tenir à la population ouvrière, outre les difficultés considérables de calcul, aurait ouvert la voie à d'autres interprétations.

3 Cf. carte, p. 349 . 
habitants et surtout les régions dont la densité communiste est inférieure à 25/10.000 babitants donnent à l'inverse les lignes de faiblesse de l'implantation communiste: l'énorme bloc de l'ouest, le gros bloc du nord-est et du centre-est jusque l'Alsace, enfin les revers oriental et méridional du Plateau central.

\section{La Stabilité relative de l'enracinement communiste}

Telle est la disposition territoriale des forces communistes en 1936 . Or, à la comparer avec celle qu'on peut établir à d'autres dates, on s'aperçoit qu'elle est à peu près constante: la profondeur de l'enracinement change suivant les périodes, tantôt diminuant, tantôt augmentant mais la disposition relative des effectifs communistes demeure. Autrement dit, entre 1921 et 1939, le réseau des organisations communistes en France ne s'apparente pas à une pieuvre qui tantôt se rétracte, tantôt se déploie, mais, telle une éponge, tantôt il se dessèche, tantôt il se gorge de forces neuves sans jamais beaucoup modifier son assise géographique.

Hypothèse qui se confirme quand on analyse une période de déclin, telle la période $1925-1933$.

\section{A. L'enracinement communiste de 1925 à 1933}

En I925, le P.C.F. est encore crédité de 55 à 60.000 adhérents. En 1933, il n'est plus crédité que de 20 à 25.000 membres. A l'échelle nationale, il a donc perdu plus de $50 \%$ de ses effectifs.

Or, si l'on compare l'implantation régionale des effectifs en 1925 et celle de 1933 , on distingue trois catégories de régions: ${ }^{1}$ $\mathrm{I}^{0}$ - Les régions dont la perte en effectifs est sensiblement égale à la perte moyenne nationale $(\approx 50 \%)$ : le Nord, la vallée du Rhône et la Région méditerranéenne, enfin la Région bordelaise.

$2^{0}$ - Les régions dont la perte en effectifs est inférieure à la moyenne nationale: la Région parisienne, le Massif Central et le Sud-ouest. $3^{0}$ - Les régions dont la perte en effectifs est supérieure à la moyenne nationale: l'Est, l'Alsace-Lorraine, la Bretagne.

I Il a fallu, bien entendu, pour rendre les comparaisons possibles dessiner des régions artificielles qui s'adaptent à la fois au dessin des structures régionales I925 et des structures régionales I933. L'étude des effectifs des régions en 1925 est fondée sur la série des nombres de cartes délivrées par région en I925, série que fournit le Rapport moral du Comité central pour le congrès de Lille (juin 1926). L'étude des effectifs des régions en I933 est fondée sur la série des nombres de timbres-contrôle placés par région en I933, série que fournit le document Quatre années de lutte pour l'unité . ., p. 42, établi pour le congrès de Villeurbanne (janv. 1936). La carte p. 349, dans ces conditions, donne, par très grands secteurs, le pourcentage des effectifs 1933 par rapport aux effectifs 1925 . 


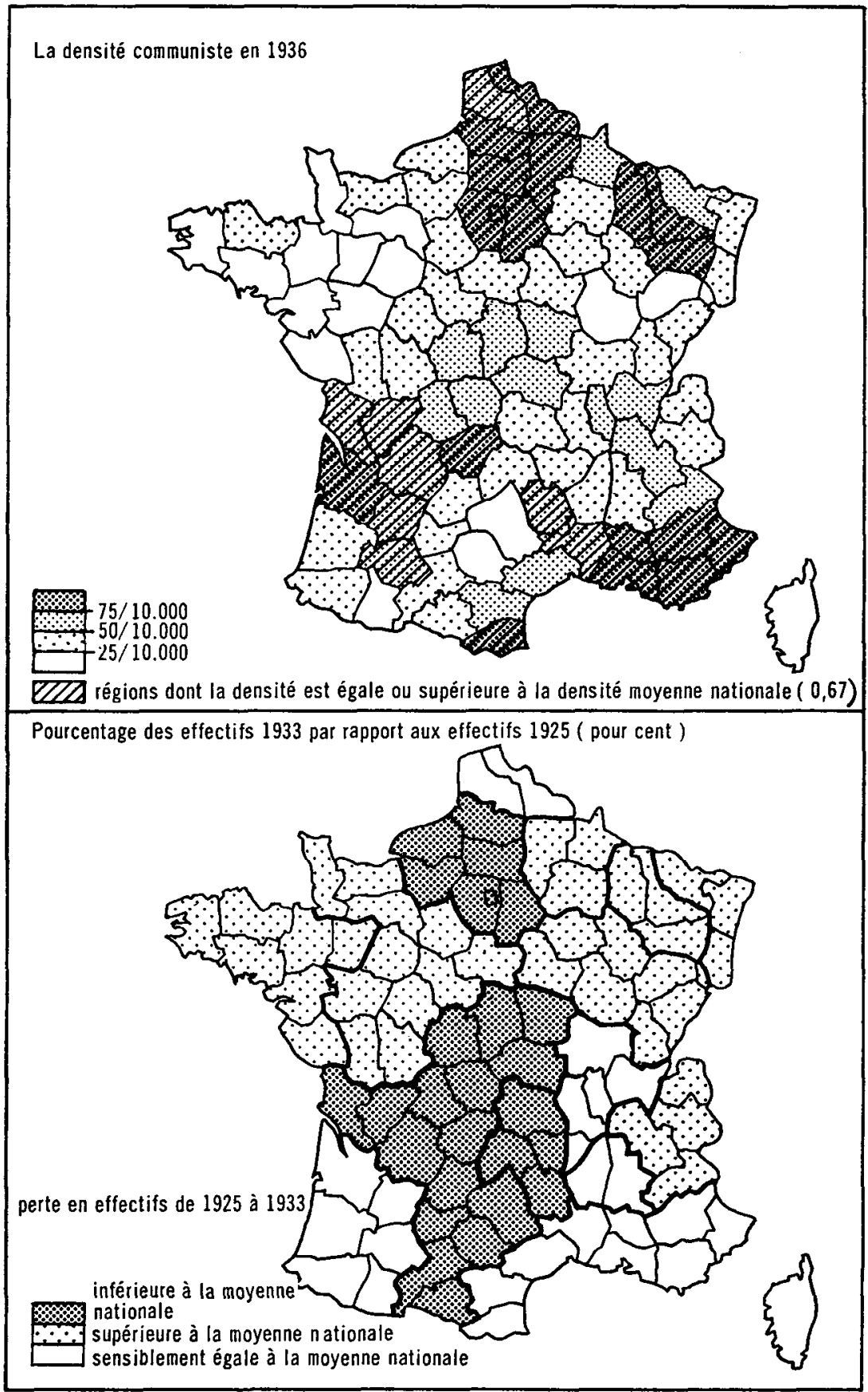


Il semble donc que résistent mieux à une conjoncture de baisse les régions de plus forte implantation (telle la Région parisienne); résistent moins bien les régions de plus faible implantation (telle la Bretagne). ${ }^{1}$ Autrement dit une période de déclin accentue encore les caractères originels de l'enracinement en augmentant l'écart entre régions fortes et régions faibles. Une exception notable, mais non surprenante: l'Alsace-Lorraine qui était une région forte et dont la perte en effectifs est très supérieure à la moyenne; il se confirme par là que l'Alsace-Lorraine, terre de contact entre des traditions socialistes différentes, exige, dans tous les domaines de l'histoire de son mouvement ouvrier, des analyses particulières. ${ }^{2}$

\section{B. L'enracinement communiste de 1935 à 1937}

On peut vérifier la même hypothèse en étudiant la période 1935-1937, période de croissance d'une ampleur exceptionnelle: en décembre 1935 , les effectifs communistes atteignent, sur la base d'un dénombrement régional, 87.752; en décembre 1936, sur la même base, 275.737; en décembre $1937,323.283 .^{3} \mathrm{Or}$, si l'on compare l'implantation des ces effectifs en hausse en $1935,1936,1937$, il apparaît: ${ }^{4}$

$\mathrm{i}^{\mathbf{0}}$ - Une stabilité étonnante de la répartition des effectifs entre les différentes régions.

$\mathrm{Si}$, en effet, on établit pour chaque fédération le pourcentage des effectifs qu'elle rassemble par rapport au total national des effectifs de l'année en cause, on peut finalement classer les 65 fédérations en quatre catégories:

(a) Les fédérations dont les effectifs représentent moins de $0,5 \%$ du total;

(b) Les fédérations dont les effectifs se situent entre 0,5 et $\mathrm{x} \%$ du total;

(c) Les fédérations dont les effectifs se situent entre i et $2 \%$ du total;

(d) Les fédérations dont les effectifs sont supérieurs à $2 \%$ du total.

Or, sur les 6 f fédérations, 41 , soit près des $2 / 3$, ne changent pas de

1 Cf. carte de la densité communiste en 1936.

${ }^{2}$ A cette date, il faut notamment tenir compte de la question des rapports du P.C.F. et du mouvement autonomiste alsacien.

${ }^{3}$ Les trois séries numériques sont empruntées au document officiel suivant: Parti communiste français, Deux années d'activité pour la renaissance économique et politique de la République française. Rapports du Comité central pour le XIème congrès national du P.C.F. Strasbourg, 25-28 juin 1947. Les circonscriptions de base sont celles de 1935-1936.

4 Cf. cartes p. 352 . 
catégorie de 1935 à 1937: leur croissance est à l'image de la croissance moyenne nationale. Ces fédérations dont la croissance est conforme à la croissance moyenne nationale appartiennent aux quatre catégories: I 6 appartiennent à la Ière; i 2 à la zème; 4 à la 3ème; 9 à la 4 ème catégorie.

$2^{0}$ - Dans le cadre de cette stabilité fondamentale, on peut dégager néanmoins une certaine tendance à la correction des facteurs bistoriques par les facteurs sociologiques de croissance.

En effet, sur les 24 fédérations qui changent de catégorie de I935 à 1937 ,

- 5 en changent de façon désordonnée: c'est le cas de la CharenteMaritime, de l'Indre-et-Loire, de l'Isère, du Puy-de Dôme, de l'Alsace (Bas-Rhin et Haut-Rhin). Seule une analyse spécifique pourrait probablement rendre compte de ce type d'anomalie.

- Io régressent d'une catégorie supérieure à une catégorie inférieure: or, il apparaît que ce sont essentiellement des fédérations d'enracinement ancien remontant, par-delà le proche passé communiste, à la plus lointaine histoire du socialisme (le plus souvent dans sa version guesdiste): ${ }^{1}$ cet enracinement ancien et déjà important ne laissait sans doute pas une marge suffisante à des possibilités de croissance correspondant au taux de croissance des années 1936 et 1937. Que la Corrèze, la Dordogne, le Lot-et-Garonne, départements pourtant essentiellement agricoles, fassent état par exemple en 1935 d'effectifs représentant de 1,37 à $3,19 \%$ des effectifs globaux, cela ne s'explique pas par des données de caractère sociologique mais par l'bistoire de l'implantation initiale du socialisme et du communisme dans ces départements. Autrement dit, leur recul relatif dans les années 1936 1937 corrige une situation qui, s'expliquant par des circonstances historiques, ne correspondait pas à ce qu'aurait dû être, dans la logique sociologique, l'implantation communiste dans ces départements de faible industrialisation.

- 9 progressent d'une catégorie inférieure à une catégorie supérieure.

On trouve là, semble-t-il, deux types de féderations. D'une part, des fédérations-Hte-Vienne-Creuse, Drôme-Ardèche, Somme-Oisequi sont des terres de vieille et forte implantation socialiste (S.F.I.O.): faut-il penser que les nouvelles générations s'y prononcent pour le communisme? D'autre part des fédérations (encore celle de SommeOise, mais aussi et surtout Loire-Atlantique, Cher-Indre, Doubs, Seine-Maritime, Meurthe-et-Moselle) où l'implantation communiste fut, pour des raisons variées, largement manquée au départ, où, par

${ }^{1}$ Ce ne peut être un hasard qu'on retrouve ici la Marne, l'Allier, le Gard et le bloc des départements du sud-ouest: Gironde, Lot-et-Garonne, Dordogne, Corrèze. 


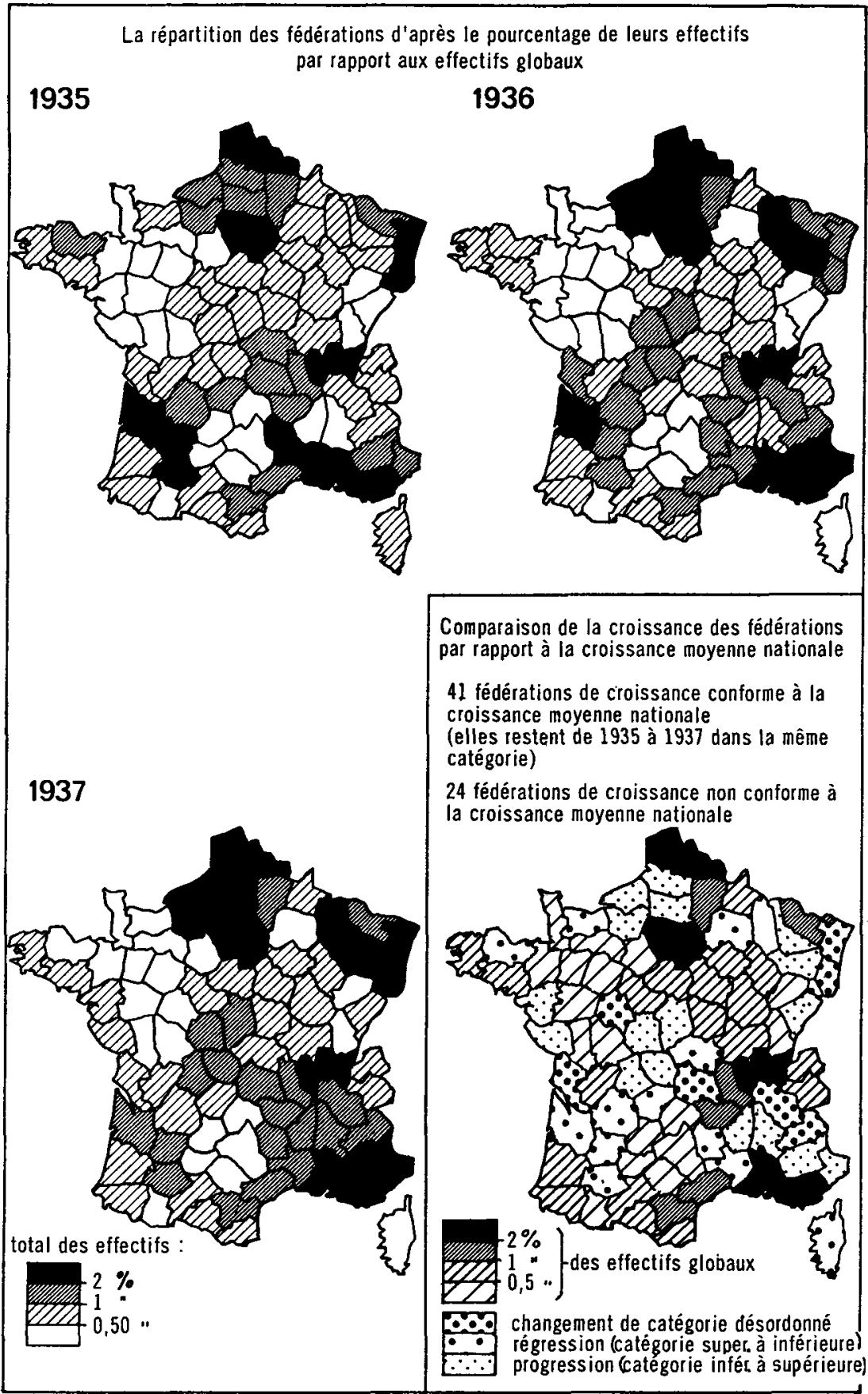


conséquent, le pourcentage des effectifs par rapport au total national était sensiblement inférieur à ce qu'on aurait pu penser compte-tenu de l'industrialisation de ces départements. Faut-il ajouter, au moins, pour le Doubs et la Loire-Alantique, que surgit ici l'hypothèse - que nous serons amenés à formuler plus loin - sur le rôle du secteur de la métallurgie dans le Front populaire?

Finalement, ce qui l'emporte, c'est la stabilité.

Comment expliquer cette remarquable stabilité qui fait que seules les situations les plus choquantes au regard du sociologique sont corrigées par une modification favorable de la conjoncture et par contre confirmées quand l'historique devient un élément de résistance dans une conjoncture défavorable?

Ne s'explique-t-elle pas notamment par les conditions dans lesquelles est né le P.C.F.: constitué par décision de la majorité du Parti socialiste, il trouva tout naturellement des adhérents partout où le parti socialiste était implanté? Or, en 1920, le mourement socialiste français, compte tenu de ses différentes nuances, avait déjà poussé de profondes racines sur la quasi-totalité du territoire national.

Mais il faut aussi faire appel à un autre élément non négligeable d'explication: la stabilité relative de l'implantation communiste est partiellement due à la mise en place d'un "appareil»" ${ }^{1}$ sur lequel, malheureusement, il est difficile de rassembler des données chiffrées. ${ }^{2}$ Ce n'est qu'à partir de 1936 qu'on peut en estimer avec quelque précision la composition. ${ }^{3}$ Par contre sa fonction est claire: maintenant en toutes circonstances ce qu'on appelle aujourd'hui une structure d'accueil, l'existence d'un tel appareil, si vainement coûteux semble-til en période creuse, est, avec ses cadres toujours prêts, la condition du succès et de l'expansion quand la conjoncture s'y prête.

\footnotetext{
${ }^{1}$ Ne doivent être considérés comme membres de «l'appareil» au sens donné à ce terme dans les pays socialistes que les fonctionnaires permanents appointés par le Parti communiste. Mais cette définition est en fait restrictive: de nombreux "permanents" sont rétribués, en théorie ou en pratique, par les trésoriers des organisations «de masse» - syndicats, coopératives, etc. - où ils occupent des fonctions responsables; ce sont néanmoins des "permanents» du parti car ils ont reçu préalablement l'investiture de la Section des cadres de la Fédération ou du Comité central communiste. Au surplus sont considérés comme membres de l'appareil tous ceux qui, élus maires ou députés, nommés à des fonctions administratives communales, professionnelles ou d'Etat où ils sont les mandataires désignés du parti, reçoivent un traitement public.

${ }^{2}$ Le parti social-démocrate allemand avait au contraire multiplié les études sur la bureaucratie socialiste et syndicale.

${ }^{3}$ Nous en donnerons une description dans notre travail sur «La formation des élites communistes" (à paraître).
} 


\section{LA RÉGION PARISIENNE}

Pour aller plus loin dans l'analyse, la France entière est un trop vaste champ d'exploration. Il faut se borner à la Région parisienne.

\section{La configuration de la Région parisienne}

De 1920 à 1924, les fédérations communistes de la Seine, de la Seineet-Oise, de la Seine-et-Marne, de l'Oise sont organisées sur la base départementale traditionnelle. En novembre 1924, dans le cadre de la réorganisation qui accompagne la «bolchevisation» du parti, la mise sur pied d'une Région parisienne est décidée, rassemblant les quatre départements. ${ }^{1}$ La première conférence de cette Région parisienne se tient en juin 1926, la seconde à la mi-juin 1927. Dans cette immense circonscription, un système complexe de parrainage est élaboré de façon à ce que les rayons les plus prolétariens apportent leur aide aux rayons dont la population est à dominante rurale. Les rayons sont d'ailleurs conçus de façon à englober quelques arrondissements ou fractions d'arrondissements parisiens, des communes ouvrières de banlieue et des communes-dortoirs, parfois même des communes purement rurales. ${ }^{2}$ Composition ingénieuse en théorie mais qui a l'inconvénient de poser de graves problèmes de communication, un même rayon ayant la responsabilité d'un territoire exagérément étiré. Aussi en février 1932 envisage-t-on de modifier la disposition des rayons: tous les arrondissements de Paris seraient regroupés en un seul rayon tandis que la banlieue serait découpée en 6 rayons. ${ }^{3}$ Mais à l'automne de cette même année 1932, le Comité central entame un processus général de décentralisation: il ne s'agit plus de modifier le découpage à l'échelon intermédiaire des rayons mais au niveau de la Région elle-même. L'immense Région parisienne de la période I924-1932 donne naissance à cinq régions: Paris-Ville, Paris-Ouest, Paris-Est, Paris-Nord, Paris-Sud. ${ }^{4}$

${ }^{1}$ Cf. Parti communiste - Région parisienne, Rapport moral et politique (1926-1927).

2 Voici par exemple, avant la réforme d'octobre 1932, la structure d'un seul rayon: I zème artt, sème arrt, Ivty, Vitry, Villejuif, Kremlin, Choisy-le-Roi, L'Haÿ-les-Roses.

${ }^{3}$ Les 6 rayons de banlieue prévus auraient eu respectivement leur siège à Irry, Boulogne, Levallois, St-Denis, Pantin, Charenton.

4 Le département de l'Oise a été antérieurement rattaché à une Région picarde dont fait partie également le département de la Somme. Le département de la Seine-et-Marne est inclus tout entier dans la Région Paris-Est. Paris-Nord, Paris-Sud, Paris-Ouest comprennent donc des communes de banlieue de la Seine et des communes de Seine-et-Oise. 


\section{Les effectifs de la Région parisienne}

En octobre 1919, les quatre fédérations départementales socialistes de la Seine, de la Seine-et-Oise, de la Seine-et-Marne et de l'Oise avaient délivré au total 26.70 I cartes. ${ }^{1}$ En octobre I921, les quatre fédérations communistes de ces mêmes départements étaient créditées de 23.535 adhérents mais en octobre 1922 , chiffre plus sûr, le nombre de leurs adhérents montait seulement à $16.225 .^{2}$ En 1925 , après la formation d'une seule Région parisienne, le point de départ est, selon A. Ferrat, de 16.674 adhérents. ${ }^{3}$ Mais à Lille, en juin 1926 , on ne fait état que de 13.586 cartes titulaires délivrées en $1925^{4}$ et même dans une conférence de la Région parisienne qui se tient uun an après Lille», soit à la mi-juin 1927, le score établi pour la fin d'année i92s est seulement de 12.600 adhérents. ${ }^{5}$ Par contre la même conférence de la Région parisienne affirme qu'en fin 1926 on pouvait tabler sur I6.824 adhérents: ce qui impliquerait que l'année I 926 fut une année de sensible redressement. ${ }^{6}$ En fait l'accroissement des effectifs de la Région parisienne entre 1925 et 1926 est sans doute dû au rattachement de la Seine-et-Marne et de l'Oise. En tout cas pour 1928 le nombre des adhérents est ramené par un rapport de 1932 à 13.472 et pour 1929 à 10.148 .7 En juillet 1930 , Thorez déclare que la Région parisienne a perdu sur l'année précédente 6.000 adhérents, ce qui ne devrait donc laisser qu'un reliquat de 4.184 adhérents: ${ }^{8}$ en fait le rapport d'organisation de février 1932 en accorde $6.895 .^{\circ}$ En $193 \mathrm{I}$,

${ }^{1}$ Parti socialiste (S.F.I.O.), Congrès national de Strasbourg (fév. 1920). Rapport du secrétariat. La vie du parti d'octobre 1918 à janvier I930, Paris, Imp. de la presse, r920, p. 93. 2 Parti communiste (S.F.I.C.), Congrès national de Paris (15-1 8 octobre 1922). L'action communiste et la crise du parti. Rapport du secrétariat général, Paris, La Cootypo, 1922, p. IOI.

${ }^{3}$ A. Ferrat, op. cit., p. I44.

${ }^{4}$ P.C.F., sème congrès national, Lille (r9-26 juin 1926). Rapport moral du C.C., in-fol., $30 \mathrm{pp}$.

${ }^{5}$ Parti communiste (S.F.I.C.) - Région parisienne, Un an après Lille. Rapport moral et politique, 1926-1927, Courbevoie, La Cootypo., s.d., in-8, i 12 pp.

${ }^{8}$ Id. p. 16.

7 Parti communiste - Région parisienne, Rapport d'organisation pour la conférence de la Région parisienne des 26,27 et 28 février 1932,34 pp., p. 5. Le chiffre de ro.148 donné pour I929 est certainement un maximum. En effet l'état de l'organisation du parti annexé au Rapport politique du Comité central (P.C.F., congrès national, 3 I mars-6 avril 1929, Paris, Bureau d'éd., I929, I02 pp.) fixe à 8.000 le chiffre des adhérents de la Région parisienne en 1929. Par ailleurs le relevé détaillé des effectifs, rayon par rayon, pour la Seine et la Seine-et-Oise a été publié dans: Région parisienne du P.C., conférence régionale, I-2 février 1930 , p. 17. Ce relevé accorde 3.804 adhérents pour la Seine (il manque les effectifs des Gème, 8ème, I I ème, I zème, I sème rayons) et $2.09 \mathrm{r}$ pour la Seine-et-Oise: soit un total de 5.895 (non compris par conséquent la Seine-et-Marne).

8 Celor, Thorez, Barbe, op. cit., p. 29.

Op. cit., p. 5 . 
Maurice Thorez annonce «s00 à 600 adhésions dans la Région parisienne», ${ }_{1}^{1}$ phénomène qui se trouve à peu près confirmé par le chiffre de 7.1 96 adhérents que reconnaît le même rapport d'organisation de février 1932. Ces 7.1 56 adhérents se répartissent de la manière suivante: 5.039 dans la Seine, 1.734 en Seine-et-Oise, 383 en Seine-etMarne. ${ }^{2}$

Quand à l'automne 1932, la Région parisienne est découpée en cinq nouvelles Régions, les cinq sont également squelettiques: au début de 1933, Paris-Ville ne compte que 2.300 membres, Paris-Est I600, Paris-Ouest moins de $1.000 .^{3}$

Suivons cette dernière Région. A la fin de l'année 1933, elle ne groupe que 1.610 membres. ${ }^{4}$ En 1934, petit démarrage: 2.352 adhérents. ${ }^{5}$ En novembre 1935 , il y a plus que doublement par rapport à 1934: 5.024 adhérents. En mai 1936, les progrès sont cependant encore modestes : 7.76I adhérents mais cinq mois plus tard, les effectifs de Paris-Ouest atteignent 25.440 adhérents. ${ }^{6}$ L'usine-pilote de la Région - Renault - abritait I 20 communistes en mai 1936 , elle en abrite 6.000 en décembre 7 . Le score final pour 1936 ne s'établit cependant qu'à 22.263. ${ }^{8}$ Pour 1937, il sera de 30.294 et pour 1938 (fin septembre) de 31.31 I. $^{9}$

\section{Le poids de la Région parisienne dans le dispositif communiste}

En étudiant l'implantation communiste en France, nous avions cru pouvoir souligner la relative stabilité du dispositif des forces communistes: sans que son assise se modifie, le Parti communiste, réagissant à la manière d'une éponge, se gonfle ou se dessèche suivant la qualité de la conjoncture.

1 Maurice Thorez, Euvres, Liv. II, t. 2, p. 56.

2 Op. cit., p. 5 .

M. Gitton, Le parti des travailleurs de France, p. 32.

M. Gitton, op. cit.

- P.C.F. - Région Paris-Ouest, sème conférence régionale (I6-17 janvier 1937), p. 31 .

${ }^{6}$ Id. Voici, en détail, la progression pour 1936 : 15 mai: $7.761 ; 18$ juin: 10.146; 8 juillet: 13.624; 30 juillet: 18.270; 15 août: 20.995; Ier octobre: 24.850 ; 10 octobre: 25.440 .

7 Id., p. 35. C'est en décembre 1936 que fut fondée la Section Remault avec ss cellules. CF. P.C.F. - Région Paris-Ouest, IVe conférence de la section Renault. Salle Lucien, Billancourt (28 mai 1938). 24 pp. En avril I93I, il n'y avait pour toute l'usine Renault qu'une seule cellule comptant 19 adhérents travaillant dans l'usine et I rattaché, soit vingt adhérents membres du i4e Rayon de la Région parisienne (cf. P.C. - Région parisienne, Rapport d'organisation pour la conférence du 26-28/2/1932, I932, 34 pp.). - Voici un autre exemple, celui d'Argenteuil, capitale ouvrière de la Seine-et-Oise: janv. 1936:425; mars: 480 ; juin: 930 ; juillet: 1330 ; août: 1355; septembre: I 505 .

8 Après retour des cartes et timbres non placés. Cf. P.C.F. - Région Paris-Ouest, 8ème conférence (novembre 1938, p. 46).

- Id. 
Cette affirmation ne doit pas être néanmoins poussée au point de considérer que chaque région garde de façon absolument constante le même poids relatif à l'intérieur de l'ensemble. Il est par exemple fort intéressant d'étudier les variations du poids relatif de la Région parisienne ${ }^{1}$ par rapport à l'ensemble du Parti.

Dans l'ancien Parti socialiste unifié, les quatre fédérations socialistes de la Seine, de la Seine-et-Oise, de la Seine-et-Marne et de l'Oise groupaient environ $20 \%$ du total des adhérents: un adhérent socialiste sur cinq habitait la région parisienne. ${ }^{2} \mathrm{Ce}$ pourcentage s'est maintenu quasi sans changement jusqu'en 1926.8 Mais la réorganisation des années 24-2s entraîne une augmentation du poids relatif de la Région parisienne: on passe à $27 \%$ en $1926,29 \%$ en I $928 .{ }^{4}$ Cela peut s'expliquer si l'on songe que la «bolchevisation», en poussant à la constitution de cellules d'entreprise, a favorisé les régions à dominante urbaine et industrielle. Au surplus, on peut se demander si le déclin général des effectifs du Parti au cours des années 1925-1933 n'a pas été légèrement freiné dans les Régions où l'enracinement était le plus profond et le plus ancien: hypothèse qu'on a déjà eu l'occasion de formuler. ${ }^{5}$ Cependant il ne pourrait s'agir que d'un freinage, car en I930-1931, le pourcentage des adhérents de la Région parisienne par rapport au total des adhérents est de nouveau de 20 à $23 \%$, c'est-à-dire à peu près conforme à ce qu'il était traditionnellement. ${ }^{6}$

\footnotetext{
${ }^{1}$ On entend par Région parisienne la circonscription qui, dans les années r926-r932 couvrait les départements de la Seine, de la Seine-et-Oise, de la Seine-et-Marne et de l'Oise, puis (au pluriel) les cinq Régions parisiennes des années 1933-I939 (Paris-Ville, Paris-Ouest, Paris-Est, Paris-Nord, Paris-Sud) couvrant les trois départements de la Seine, de la Seine-et-Oise et de la Seine-et-Marne. On remarquera que la Région parisienne des années 1926-1927 est plus étendue que les cinq Régions parisiennes des années 1933-1939, puisqu'elle englobe l'Oise, laquelle fut assez vite détachée de la Région parisienne pour former avec la Somme une Région picarde. Cette légère et éphémère différence ne modifie pas le raisonnement qui va suivre.

${ }_{2}^{2}$ Au 31 décembre 1919, les quatre fédérations socialistes rassemblaient en effet $26.70 x$ adhérents sur 133.327, d'après le Rapport du Secrétariat au congrès national de Strasbourg (fév. 1920).

${ }^{8}$ En 1921, les quatre fédérations communistes rassemblaient 23.535 adhérents sur 109.391, soit $21 \%$; en 1922 I6.225 sur 78.828, soit $20 \%$. D’après les états publiés dans P.C. (S.F.I.C.) Congrès national de Paris. L'action communiste et la crise du Parti, p. ror. En 1925, la Région parisienne nouvellement constituée est créditée (au congrès national de Lille, Rapport moral du Comité Central) de I3.586 cartes titulaires délivrées. Par rapport au total des effectifs $-60.000-$, elle représente $22 \%$.

4e Rapport moral du Comité central à Lille (juin I926) accorde I 5.000 adhérents (cartes titulaires délivrées au is avril) pour 55.000 adhérents (P. Sémard au congrès de Paris-StDenis en 1929). En 1928, la Région parisienne compte 13.472 adhérents (Rapport d'organisation, fév. 1932, p. s) pour un total (approximatif) de 45.000 (cf. p. 359).

5 Cf. p. 348.

- Les effectifs des cinq Régions parisiennes sont en 1933 de 9.080 (sur un total de 28.754,
} 
Mais, à partir de I933, après la décentralisation de la Région parisienne en cinq Régions, le poids de l'ensemble des cinq Régions par rapport au parti tout entier, va considérablement augmenter: $3 \mathrm{I} \%$ en $1933 ; 34 \%$ en $1934 ; 33 \%$ en $1935 ; 36 \%$ en $1936 ; 35 \%$ en 1937 ; $32 \%$ en 1938 . Ces pourcentages confirment bien ce qu'écrivait en 1936 La Correspondance Internationale: le tiers des adhérents se trouvait dans la région parisienne. ${ }^{1}$

La question se pose alors de savoir si la croissance très rapide des effectifs du Parti favorise les grandes régions déjà bien enracinées et si, par conséquent, l'augmentation du poids de la Région parisienne par rapport à l'ensemble n'est qu'un corollaire quasi-obligatoire de la croissance générale.

Il n'en est rien. En effet, si on calcule le poids des Fédérations de la Seine, de la Seine-et-Oise et de la Seine-et-Marne par rapport au Parti tout entier dans cette autre période de croissance remarquable que furent les années 1945-1946, on retrouve les pourcentages habituels: I7 et $18 \%{ }^{2}$ Autrement dit, en 1945-1946, moins d'un communiste sur cinq habitait la Région parisienne: en 1936, plus d'un sur trois. Ce constat ne saurait en lui-même permettre des conclusions. Mais il encourage à former une hypothèse: la Résistance - pas seulement pour des raisons techniques liées au type très spécial de guerilla qu'elle implique - fut pour beaucoup un phénomène provincial, un phénomène décentralisé qui, par conséquent, mit en mouvement et bouleversa les régions reculées du pays. Le Front populaire au contraire ne fut-il pas, sinon exclusivement, du moins fortement parisien? Il $\mathrm{y}$ aurait là une direction de recherche que les chiffres suggèrent mais dans laquelle il faudrait s'engager avec une information autre qu'une information seulement numérique.

Cette hypothèse nous a du moins conduit à deux observations qui seraient de nature à la confirmer.

$\mathrm{Si}$ on isole la Région Paris-Ville et qu'on compare ses effectifs à ceux de l'ensemble des cinq Régions parisiennes, on constate que, de 1933 à septembre 1938 , les effectifs de Paris-Ville ont exactement augmenté dans la même proportion que l'ensemble des effectifs des

cf. p. 359); en 1934 de 14.283 (sur un total de 42.000); en 1935 de 28.860 sur un total de 87.752; en $x 936$ de 99.838 sur un total de 275.737 ; en 1937 de $\times 15.367$ sur un total de 323.283 ; en 1938 de 104.845 sur un total de 318.549 . Cf. les séries numériques extraites des Rapports du Comité central publiés en vue des congrès de Villeurbanne (janvier r936), d'Arles (décembre 1937), de la conférence nationale de Gennevilliers (janvier 1939), des congrès de Paris (mars I945) et de Strasbourg (juin 1947).

$1 \mathrm{~J}$. Berlioz, La Correspondance internationale, 18 juillet 1936, p. 875 . Même affirmation dans M. Gitton, Le grand parti du peuple de France, p. 4 (r I juillet r936).

2 En 1945, 138.652 adhérents dans les trois fédérations de la Région parisienne pour un total de 775.342; en 1946 , 147.448 pour un total de 804.229. 
Régions parisiennes. Le poids de Paris-Ville s'est situé de façon constante entre 28 et $32 \%$ de l'ensemble des cinq Régions parisiennes. ${ }^{1}$ Bref, de façon constante, entre 1933 et 1938 , moins d'un communiste sur trois militants dans une des cinq Régions parisiennes, était organisé dans le cadre de Paris-Ville. Ce qui est largement inférieur au pourcentage de la population de Paris par rapport à la population globale des trois départements concernés. ${ }^{2}$

Au contraire, si l'on isole la Région Paris-Ouest et qu'on compare ses effectifs à ceux de l'ensemble des cinq Régions parisiennes, on constate que, de 1933 à septembre 1938 , les effectifs de Paris-Ouest ont augmenté dans une proportion beaucoup plus forte que l'ensemble des effectifs des Régions parisiennes. Le poids de Paris-Ouest n'a cessé de croître: représentant $14 \%$ du total des effectifs des cinq Régions parisiennes en 1933, Paris-Ouest en représente successivement $16 \%$ en $1934,18 \%$ en $1935,27 \%$ en $1936,29 \%$ en $1937,30 \%$ en 1938.3

Observation complémentaire: les cinq courbes représentant la croissance des effectifs dans les cinq Régions parisiennes de 1933 à 1938 soulignent bien que la croissance de la Région Paris-Ouest a été beaucoup plus forte que celle des Régions Paris-Sud, Paris-Est et surtout Paris-Nord. ${ }^{4}$

1 Voici le tableau permettant de calculer le poids relatif des effectifs de Paris-Ville par rapport au total des effectifs des 5 Régions parisiennes.

$\begin{array}{cccc}\text { Année } & \text { Paris-Ville } & \text { Total Rég. parisienne } & \% \\ 1933 & 2.884 & 9.080 & 31 \% \\ 1934 & 4.575 & 14.283 & 32 \% \\ 1935 & 8.450 & 28.860 & 29 \% \\ 1936 & 31.560 & 99.838 & 31 \% \\ 1937 & 34.530 & 115.367 & 29 \% \\ 1938 & 30.000 & 104.845 & 28 \%\end{array}$

${ }^{2}$ Au recensement de 1936 , la population de Paris constitue $41 \%$ de la population des trois départements.

$\begin{array}{cccc}3 \text { Année } & \text { Paris-Ouest } & \text { Total Rég. parisienne } & \% \\ 1933 & 1.300 & & 14 \% \\ 1934 & 2.372 & & 16 \% \\ 1935 & 5.400 & & 18 \% \\ 1936 & 27.048 & \text { Cf. p. 034, note I. } & 27 \% \\ 1937 & 34.44^{8} & & 29 \% \\ 1938 & 31.550 & & 30 \%\end{array}$

4 Sur la base des effectifs suivants:

$\begin{array}{cccccc}\text { Année } & \text { Paris-Ville } & \text { Paris-Ouest } & \text { Paris-Nord } & \text { Paris-Est } & \text { Paris-Sud } \\ 1933 & 2.884 & 1.300 & 1.676 & 1.660 & 1.560 \\ 1934 & 4.575 & 2.372 & 2.436 & 2.824 & 2.076 \\ 1935 & 8.450 & 5.400 & 4.235 & 5.950 & 4.825 \\ 1936 & 31.560 & 27.048 & 8.500 & 15.500 & 17.230 \\ 1937 & 34.530 & 34.448 & 10.889 & 16.500 & 19.000 \\ 1938 & 30.000 & 31.550 & 10.095 & 15.700 & 17.500\end{array}$


Comment expliquer le fait? L'hypothèse la plus séduisante me semble celle-ci: c'est la composition très prolétarienne de la Région Paris-Ouest qui explique qu'entre toutes les Régions parisiennes la croissance de celle-ci fut à l'époque de beaucoup la plus forte.

Encore faut-il préciser par quels mécanismes. La Région ParisOuest est en effet une circonscription où les localités dont la population est bourgeoise ou petite bourgeoise en majorité l'emportent sur les localités dont la population est nettement ouvrière. On y trouve Neuilly, Boulogne, St-Germain-en-Laye, Maisons-Laffitte, Marly-le-Roi, Versailles, Rambouillet et des cantons tout à fait ruraux (Houdan, Magny-en-Vexin, etc.).

Mais c'est oublier que les structures d'organisation du Parti communiste rejettent, chaque fois que cela est possible, la base locale pour adopter la base de l'usine, de l'entreprise. Or, si la Région Paris-Ouest a une population peut-être plus aisée en moyenne que les autres Régions parisiennes - les communes administrées par des municipalités communistes y sont peu nombreuses -, en revanche elle est par excellence une zone industrielle où des dizaines de milliers d'ouvriers se rendent chaque jour: les industries de l'automobile, de l'aviation, de la métallurgie différenciée connaissent à Billancourt, Puteaux, Clichy, Levallois, Gennevilliers, Poissy un large développement.

C'est cette infrastructure industrielle de la Région Paris-Ouest, de loin la plus forte de toute la Région parisienne, qui explique l'extraordinaire croissance de ses effectifs dans les années I933-1938.1 On peut encore le vérifier d'une autre manière: le pourcentage du nombre des cellules d'entreprise par rapport au nombre total des cellules implantées dans la Région Paris-Ouest passe de $4 \mathrm{r} \%$ en mai 1934 à $54 \%$ en novembre 1936. C'est donc l'adhésion d'ouvriers et la constitution de source de la croissance. ${ }^{2}$

${ }^{1}$ C'est ici qu'on voit combien les analyses strictement électorales ne permettent pas d'induire ce qu'il en est de la communauté partisane à partir de la communauté électorale. Les municipalités communistes sont concentrées dans les secteurs nord-nord-est et sud-sud-est de la Région parisienne: c'est là que se concentre l'habitat ouvrier. Mais les zones d'habitat ouvrier ne coïncident pas toujours avec les zones industrielles. Les Régions communistes d'habitat ouvrier sont donc, en fonction du principe d'organisation qui régit les militants, moins développées que les Régions communistes d'économie industrielle.

2 Voici, d'après la sème conférence régionale Paris-Ouest (16-17 janvier 1937), p. 35, et la 8ème (19-20 nov. I938), p. 44, l'évolution du nombre des cellules d'entreprise par rapport au nombre total des cellules de la Région Paris-Ouest:

$\begin{array}{cccc}\text { Année } & \begin{array}{c}\text { Cellules } \\ \text { d'entreprise }\end{array} & \begin{array}{c}\text { Cellules } \\ \text { toutes catégories }\end{array} & \begin{array}{c}\% \text { des cellules } \\ \text { d'entreprise }\end{array} \\ \text { Mai I934 } & 48 & 115 & 41 \% \\ \text { Fév. I935 } & 63 & 176 & 35 \% \\ \text { Nov. I935 } & 87 & 274 & 31 \%\end{array}$


D'où cette hypothèse à vérifier: le Front Populaire dont il nous a semblé tout à l'heure qu'il faudrait peut-être y voir un phénomène largement parisien, ne fut-il pas aussi un phénomène social plus caractérisé qu'on ne le pense parfois, un mouvement typiquement ouvrier et, de façon encore plus précise, un mouvement de métallurgistes? ${ }^{1}$

$\begin{array}{rlll}\text { Mars } 1936 & \text { IIs } & 327 & 35 \% \\ \text { Nov. 1936 } & 354 & 692 & 51 \% \\ 1937 & 464 & 844 & 54 \% \\ 1938 & 454 & 843 & 53 \%\end{array}$

1 Il nous semble qu'on ne saurait trop, du point de vue du «leadership ouvrier», refléchir sur ce fait souvent reconnu, discuté et commenté dans les cercles où se perpétuent les traditions et les souvenirs des luttes ouvrières: trois grandes explosions sociales secouèrent la France des 40 premières années du siècle; à la première, celle des années I 906-I909, les travailleurs du Bâtiment imprimèrent leur marque; à la seconde, celle des années rg I91920, les cheminots; à la troisième, les métallos. Objectifs, style, tésultats et conséquence, chacune de ces trois coxporations a donc successivement contribué à façonner le mouvement ouvrier français. 